\title{
Gray's Paradox and Wave Solutions in Explaining Anomalously Low Friction and Wear in Tribology
}

\author{
V. M. Stadnichenko, O. M. Troshin \\ Department of Hydraulic Systems, Air-Space Institute, National Aviation University, Kiev, Ukraine \\ Email address: \\ vstadnichenko@bk.ru (V. M. Stadnichenko)
}

\section{To cite this article:}

V. M. Stadnichenko, O. M. Troshin. Gray's Paradox and Wave Solutions in Explaining Anomalously Low Friction and Wear in Tribology. International Journal of Materials Science and Applications. Vol. 5, No. 1, 2016, pp. 23-30. doi: 10.11648/j.ijmsa.20160501.14

\begin{abstract}
The results of theoretical and experimental studies of the anomalously low friction are presented in this paper from the standpoint of thermodynamics of non-equilibrium processes, arising under conditions of elastic interaction of microrelief of tribosystem elements. The dependences of acoustic emission parameters which correspond to the alteration of energy dissipation mechanism of friction pairs superficial layers are obtained. In addition to molecular and mechanical components of the force of friction at anomalously low wear a wave component appears, and in this case the effect of this component prevails.
\end{abstract}

Keywords: Tribosystem, External Friction, Internal Friction, Kinetic Nanofield, Progressive Wave, Anomalously Low Friction, Acoustic Emission

\section{Introduction}

Actual progress in the development of structure-energetic [1] and molecular-mechanical [2] theories of friction and wear allow considering a tribosystem as a generator that transforms mechanical energy into heat energy. Thus, the results of calorimetrical researches $[1,3]$ prove that over $95 \%$ of energy supplied to a tribosystem is turned into heat, and about $5 \%$ of this energy is accumulated in a surface layer and then used for structural transformations, and, partly, dissipates through the disruption channel that is followed by acoustic, electromagnetic and other kinds of emission [4].

All the measures aimed at the increasing of tribosystem wear resistance, are aimed, directly or indirectly, at the increasing of efficiency of the heat generator, and two principle lines of research have been formed in this sphere. The first one consists in diminishing the part of energy which is spent to destruct the surface layer due to the use of more effective lubricants, perfect material structures, as well as application of different wear resistant coating [1]. The second one consists in perfecting conditions of contact interaction, in decreasing friction coefficient and, as its consistence, in decrease of the absolute value of external energy supplied to the tribosystem $[5,6]$.

\section{Analysis of Principal Findings and Publications}

The force of external friction is directed against motion in a tribosystem, and, in accordance with molecular-mechanical theory of wear friction (summarized by I. V. Kragelskiy), consists of two components: molecular (adhesive) $F_{a}$ and mechanical (deformation) $F_{d}$ [2]. These components of the force of friction $F$ "conflict" with each other when solid bodies move,

$$
F_{f r}=F_{a}+F_{d}
$$

When smoothing a surface using the methods of finishing treatment, the mechanical component of the force of friction may be lowered significantly. However, the molecular component increases proportionally, and at the wearing-in stage, it initiates the adhesive-cohesive destruction of the surface layer of the tribosystem. Due to the wear-in process, an equilibrium state is forming, which corresponds to the given conditions of friction (load, sliding speed, temperature, etc.). In its turn, it determines the amount of external energy which is supplied to the tribosystem, and, consequently, determines the rate of formation and destruction of secondary structures [1] on friction surfaces, that is, their wear resistance.

The summary of a large number of the results of boundary friction study [7] proves that the value of the boundary friction coefficient for different combinations of lubricating materials, in accordance with Gersi-Stribeck curve is within the range $0,005 \ldots 0,02$.

\section{Goal of Research}

Therefore, at frictional modes and with lubricants which 
are mostly used, both the surface layer of tribosystem elements and lubrication medium participate in the formation of the frictional force. At present, there are some scientific papers that demonstrate the results of experimental study when the values of the parameters of the frictional force go out of the minimum limits and are small [8,9]. For this reason, the search of the theoretical prerequisites to change external friction from "normal range" to anomalously low friction is well-timed and crucial task, and the present paper is dedicated to its solution.

\section{Description of the Principal Information}

While analyzing this problem, it is highly interesting to mention the research made by English scientist James Gray, who in 1930s stated that the reserve of muscle power of a dolphin equals only $10 \%$ of the estimated power it needs to move at the observed speed $(10 \mathrm{~m} / \mathrm{s})$.

In other words, according to the laws of hydrodynamics, a dolphin cannot move in the water at such a speed due to small reserve of its muscle power, however, it moves, and this is the essence of so called "Gray's Paradox".

During the years that passed, many hypotheses were proposed to explain the low resistance of dolphin's motion; their idea is based on the elasticity of dolphin's skin. But experimental hydrodynamic studies carried out in this sphere, gave negative results. James Gray himself helped invaluably to reveal the physical mechanism of anomalously low friction at dolphin's motion. He assumed hypothetically that a negative gradient of a hydrodynamic pressure somehow appears and acts between the surface of dolphin's nose and water from ahead, that is, a moving dolphin seems to hurry after the water from ahead that continuously runs away from it.

To realize such an interaction of objects that move in liquid, authors of published work [10] have developed a technology, whose essence consists in the fact that a decrease in hydrodynamic resistance of moving vessels is caused by formation of waves of elastic compression from the surface of ship's head in the opposing water. The realization of this approach in modern ship-building industry makes possible to increase the rate of sailing more than twofold when using actual engine power and with the same consumption of fuel.

The authors of published works [11, 12] have obtained the analogous result concerning the decrease in the force of friction, when developing the technology of tribotechnical recovery. In this case, the effect of anomalously low wear and friction was achieved due to mobility of a two-layer metal-ceramic layer formed on frictional surfaces, and it is in these works that the wave approach was used for the first time to explain the process of dissipation of energy supplied from an external source.

Without any doubt, it is practically impossible to use the said technologies to achieve anomalously low friction for the majority of real moving tribosystems operating under boundary friction conditions, when the difference between hydrodynamic and external friction is big. However, the wave nature of this phenomenon (Gray's Paradox) gives leave to propose a scientific hypothesis which states that in addition to the molecular and mechanical components of forces of friction, the wave component, $F_{w}$, also exists:

$$
F_{f r}=F_{a}+F_{d}-F_{w}
$$

Under the standard conditions of boundary friction, the surface layer "softening" of triboelements (Rehbinder effect) takes place due to interaction of a lubricating medium with triboelements' materials that are plastically deformed [7]. The positive depth gradient of the hardness of the surface layer is formed. Hence, the tribosystem changes from the elastic contact to the elastically plastic and plastic contact. At that, the force of friction decreases to a certain stationary value. At given conditions of contact interaction in the tribosystem, the numerical value of the force of friction is determined by two first components of expression (2). The wave component $F_{w}$ is negligibly small in this case, and by this reason, up to the present time it haven't been taken into account, though the authors mentioned the wave component both in past and at present. Indeed, I. V. Kragelskiy [2], while explaining the elastic deformation of a material at friction, demonstrated that the contacting points cause the elastic deformation of a material, and the unlocked contacts start to oscillate, it means that, in practice, a wave component of the force of friction is under formation [2].

The findings of the study of elastic contact at friction, given in published works $[13,14]$, prove that the oscillations under certain conditions may serve as an additional channel of dissipation of energy supplied to the tribosystem.

Conditions of formation of real tribosystems operating exclusively in the elastic range are given in published works $[7,14,15,16]$.

The theoretical basis for using wave effects to achieve anomalously low friction and wear is A. I. Veinik's published work [13], where the author, basing on the thermodynamics of non-equilibrium processes, formulated conditions needed to achieve both zero and negative external friction.

Making analysis of reversible and irreversible processes, the author of this paper came to the conclusion that the numerical value of the work of the force of friction may be considered as the criterion for process irreversibility assessment ("irreversibility coefficient"). The concept of the irreversible process at friction is extremely conventional because the irreversibility, since Clausius, is interpreted as the strict onedirectional flow of all real processes. In reality, according to A. I. Veinik, the processes with negative friction occur widely in the nature, just as the processes with positive friction.

While considering the interaction of microroughnesses in the contact zone of the tribosystem similarly to the example given in [13], the effect of transformation of mechanical energy into heat energy $Q_{D}$ in the tribosystem at elastic interaction of microroughnesses, if the interacting masses (which are microroughnesses on the surface) of the movable and immovable friction bodies are equal, may be estimated as follows:

$$
Q_{D}=(1 / 2) \delta P_{m D^{m}}=(1 / 2) m\left(\omega_{c}^{2}-\omega^{2}\right),
$$

here $\delta P_{m D}$ is the difference of kinetic energies of interacting 
microroughnesses; $m$ is the acquired (lost) mass of microroughnesses; $\omega_{c}$ is the initial velocity; $\omega$ is final velocity.

At elastic interaction of microroughnesses $Q_{D}$ depends on the grade of perfection of the interaction, which is estimated as the value of losses caused by plastic deformation or destruction. In case of absolutely elastic interaction of bodies with immovable obstacle, the velocity of the mass before interaction equals its velocity after interaction. Deceleration of the mass is accompanied by emission of dissipation heat $Q_{D}$, and its acceleration, until it gains the previous velocity, is accompanied by absorption of the same amount of heat $Q_{D}$. As a result, the screening (dissipation) effect becomes zero. It results from (3), because after substitution of the total flow of the kinetic component of the total energy of the body it becomes zero. However, the presence of the difference of squared velocities in (3) evidences that the question of which one of the velocities, initial or final one, is bigger, is extremely important. If the initial velocity $\omega_{c}$ is bigger than the final velocity $\omega$ after collision, in other words, if the activity of the kinetic component of the total energy of the body decreases due to the screening effect, then the dissipation heat is positive and it is emitted (positive friction). If the initial velocity $\omega_{c}$ is less than the final velocity $\omega$, in other words, if the activity of the kinetic component of the total energy of the body increases due to the screening effect, then the dissipation heat is negative and it is absorbed (negative friction).

In view of the above, the energetics of the surface does not allow considering the roughness only from the geometrical standpoint. An interesting physical effect of formation of Benard cells [17] is known in the theory of dissipation. If the energy is continuously supplied to the surface and the amount of this energy reaches certain value, then more intensive external energy output (dissipation) is needed. Bifurcation with the consecutive self-organization of surface structure takes place, at the same time the surface spontaneously breaks into individual elements, and each of them dissipates more intensively then before the bifurcation [18].

Let us analyze the energy exchange between wearing surfaces by representing roughnesses as energy cells, each of them gives its energy to the counterbody and receives energy from the conterbody. This energy exchange shall be estimated with surface-averaged energy flows. If we consider these flows locally, then, depending on elastic-plastic characteristics of triboelements the conditions of positive and negative friction shall be also achieved, with account of proportion between molecular-mechanical and wave components of the force of friction.

So, if the real tribosystem is represented as an assembly of interacting elastic microroughnesses, then the principal reason of minimization of values of tribological parameters (wear rate, temperature in the contact zone, force of friction, etc.) is the kinetic nanofield (reaction of masses of microroughnesses on external action that appears in local points of the elastic interaction of friction bodies). The conditions when the two effects, that of dissipation and antidissipation, become exactly equal to each other, shall be possible, because the work of forces of the nanofield always equals the work needed to decelerate or accelerate masses, however, the signs of these works are opposite.

The amount of the dissipative component $d \Theta_{D}$ which is emitted or absorbed heat, is proportional to the change in the activity of the nanofield kinetic component $d Q_{D}$, and amount of the transferred heat caused by local interactions $d E$, and inversely proportional to the absolute temperature $T$ of the carrier, which may be described by stochastic equations whose final solutions may be represented [18] as:

$$
d \Theta_{D}=d Q_{D} / T=-d P_{D} d E / T .
$$

Nowadays, to estimate a stress state of the surface layer, the level of strains of certain sings is used which cause compressive or lengthening effect in the whole effective volume of the interaction and are equilibrated in the section of contacting bodies.

In case of the kinetic nanofield, the value of local strains may be represented by the effect of liquid cavitation, when microzones with high and low pressure exist simultaneously, and the resulting value of the strain is not stationary. At realizing these conditions in a tribosystem, the total change in temperature, force of friction and wear at a load change in the zone of contact will approach zero.

Therefore, the hypothesis of quasi-elastic interaction of surfaces accompanied by effects of zero friction and negative friction proposed in published works [6, 13], comprises prospects for further development both in the theory and in solution of the principal applied problem of minimization of friction and wear for the wide range of machinery and mechanisms tribosystems.

The existence of effects of friction and anti-friction, dissipation and anti-dissipation makes us to look with a fresh eye at the problem of irreversibility of processes in tribosystems, and develop a fundamental approach on this basis to diminish friction in and wear of real tribosystems [19]. The self-regulation of tribosystems of this sort is realized through the channel of excess production of entropy whish determines the tribosystem stability in conditions of non-equilibrium self-organization [17]. A change in increment of excess entropy $\left(\delta^{2} S\right)_{m c}$ with time for nonequilibrium processes described above, has the oscillatory type. Hence, the sign will determine the proportion of dissipation and anti-dissipation processes.

In addition to kinetic interactions which appear on submicroscopic and finer levels, the behavior of surface layer after stoppage of the tribosystem is also very important for the analysis of processes of the tribosystem self-organization. It follows from published work [20] that the tribosystem stoppage results in exclusion of the boundary layer disorganization, however, the processes aimed at selforganization continue during certain time, which causes the better ordering of frictional contact and, consequently, decrease in the force of friction during the next loading. This circumstance allows to significantly change the methodology of program loading [15] to shift the tribosystem to the higher level of self-organization when it leaves the stability limits. The program loading provides for observance of complete adequacy between the growth of applied load and rate of 
relaxation of internal strains owing to diffusive and micro-shift processes. After program loading a metal becomes more homogeneous with respect to the distribution of strains. The structure of program-resistant metals is, mechanically, more equilibrated compared with the initial material, it demonstrates higher resistance against the initial plastic deformation and, at the same time, it is less prone to brittle fracture [15].

However, the impulse loading applied to the tribosystem which is in a certain structural state, results in a spontaneous transition of plastic deformations from a substructural microlevel to a meso-level that significantly exceeds the depth of a cold-work layer, and, at the same time, abrupt changes in the properties of materials of the tribosystem are observed. A quasi-elastic layer is formed on the surface, and in its central part, hypothetically, a hydrodynamic deformation is possible, while in the transition zone at the periphery an intensive rotational elastic-plastic deformation is expected to appear, which is analogous to the structure of eddy formation in the near-wall layer when a liquid flows [21].

Basing on the works by I. Prigozhyn, A. I. Veinik in the sphere of non-equilibrium self-organization, we carried out two series of experimental studies. Their general idea is to realize the wearing-in at the first stage of tribosystem operation through the achievement of the conditions of equilibrium self-organization in the tribosystem, which are characterized by constant values of average surface temperature, force of friction and wear rate. At the second stage, after the energy supply, the tribosystem passes over the stability limit. These conditions were created owing to impulse (program) loading (Fig. 1) [6].

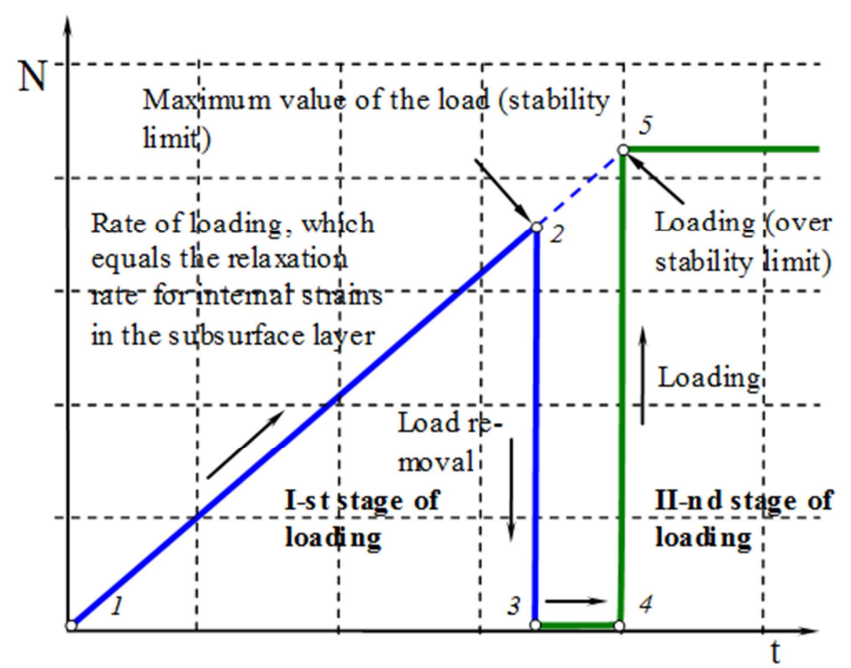

Fig. 1. Program loading when the tribosystem passes to the higher level of non-equilibrium self-organization: 1 - beginning of loading; 2 - stability limit for tribosystem transition to its damage; 3, 4-getting the maximum uniform distribution of strains in the surface layer; 4 -impulse loading; 5 bifurcation point for the tribosystem transition to the highest level of selforganization.

With the purpose to carry out the program loading, the friction machine 2070 CMT-1 was modernized to get the possibility to regulate the rate of loading within the wide range of values and carry out the impulse loading of the tribosystem [22]. Specimens of steel 30X3BA and bronze ВБ23НЦ were studied. Aviation kerosene TC-1 was used as machining medium, its flow rate equaled $3,5 \mathrm{~L} / \mathrm{h}$. Testing conditions were: the load at operation in stationary conditions $-600 \mathrm{~N}$; the angular frequency of the transmission shaft $500 \mathrm{~min}^{-1}$; the environment temperature $+20^{\circ} \mathrm{C}$.

The program loading was carried out in accordance with recommendations given in published work [6]. The quality of surfaces of the specimens prepared for testing, was high. The wear rate was measured in real time using the acousticemission method [19]. The average wear rate (the ratio of integral value of the averaged power of acoustic emission and number of measurements) was used for comparative estimation of testing tribosystems wear. The results of testing the basic tribosystem and tribosystems that experienced program loading are given in Fig. 2-4.

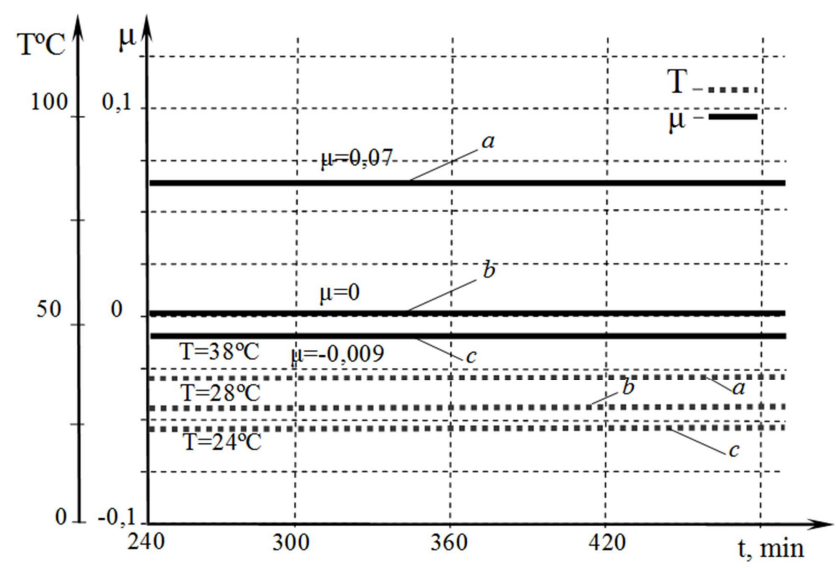

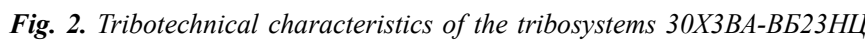
that undergone the test at the final stage of operation with the load of $600 \mathrm{~N}$ : $a$ - the basic tribosystem; $b$ - at program loading (impulse loading equals $1400 \mathrm{~N}$ ); $\mathrm{c}$-at program loading (impulse loading equals $1800 \mathrm{~N}$ ).

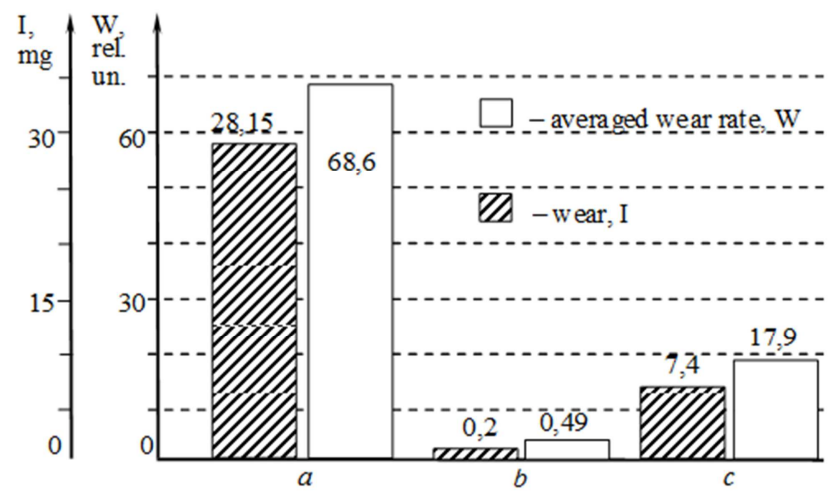

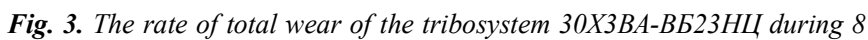
hour testing: $a$-basic tribosystem; $b$-at program loading (impulse loading equals $1400 \mathrm{~N}$ ); $\mathrm{c}$-at program loading (impulse loading equals $1800 \mathrm{~N}$ ).

The test program provided for basic testing of wear resistance with determination of principal tribological parameters when load equaled $600 \mathrm{~N}$. The second series of experiments provided for the wearing-in at a given load and tribosystem transition from the equilibrium to nonequilibrium friction as a result of program loading. According to the program, the value of the impulse load equaled $1400 \mathrm{~N}$ and $1800 \mathrm{~N}$, and then the operation load of $600 \mathrm{~N}$ was used at the final stage of loading.

The analysis of the results proved that the changeover of the tribosystem operation to the conditions of anomalously low friction and wear is characterized by the sufficiently 
good reproducibility. The results of the experimental study of friction couples 30Х3ВА-ВБ23НЦ with aviation kerosene $\mathrm{TC}-1$ used as machining medium are given in this paper.

Let us analyze the results of anomalously low friction obtained in the course of program loading with impulse loading up to $1400 \mathrm{~N}$, Fig. 1-3, b, from the standpoint of the local kinetic contact interaction in the tribosystem. In other words, let us analyze the physics of kinetic nanofield formation in case of elementary elastic contact of microroughnesses in the presence of external friction.

The most natural way to explain the interaction of effective volume of microroughnesses consists in its representation as Markov process with two discrete states, in particular, "Double-type reaction", that provides for the possibility of the sole transition in the system (state $1-x_{k}$

recovery, state $2-x_{j}$ failure), $1 \leftrightarrow 2$ (for example, deformations and recoveries of the initial shape of the effective volume).

For the said process A. N. Kolmogorov equation [23] is true:

$$
\frac{\partial p_{1}\left(t_{1} x_{k}\right)}{\partial t}=\sum_{i} A_{j k}(t) p_{1}\left(t_{1} x_{j}\right),
$$

here $p_{1}\left(t_{1} x_{k}\right)$ is the one-dimensional probability of the state; $A_{j k}=\left[\frac{\partial p\left(u_{1} x_{k} / t_{1} x_{j}\right)}{\partial u}\right]_{u=t} ; p\left(u_{1} x_{k} / t_{1} x_{j}\right)$ is the probability of transition from state $x_{k}$ to state $x_{j}$, hence, with regard to the normalization, $A_{j k}(t) \geq 0(j \neq k)$; $A_{i j}(t)=-\sum_{k \neq 1} A_{j k} \leq 0 ; \sum_{k} A_{j k}(t)=0$.

With regard to the set of local interactions of microroughnesses considered both in theoretical and experimental aspects, the probability to find an elementary contact in one of the states of the two-stage model depends on the last state at the moment $t_{n}$ and is independent of the background. The solution of the earlier formulated equation is the diffused Markov process. It is obvious that to solve this equation it is needed to impose certain limitation on its right side, in particular, assume that the loading vector $q(t)$ is time delta-correlated process. This condition is met while carrying out the experimental studies using the program loading.

Under impulse exposure at the final state of the program loading a super-powerful specific concentration takes place at micro-contacts that change material to the "magmaplasma" state [24] with specific properties.

In this case the conditions of non-equilibrium selforganization are accompanied by processes which are incompatible with traditional principles of the thermodynamics of equilibrium systems; in particular, in this case the state of the entropy self-regulation appears which is caused by processes of fluctuation symmetry in accordance with the principles of the least action [17].

Introducing the designations $p_{1}(t, 1)=p_{1}(t)$ - probability of existence of state 1 (recovery) in the system, and assuming that the process is time-uniform, and $\alpha, \beta$ are constants: $A_{12}=\alpha ; A_{21}=\beta ; \alpha+\beta=\lambda$, here $\alpha \partial t ; \beta \partial t$ are the probabilities of transitions $1 \leftrightarrow 2$ (failure) and $2 \leftrightarrow 1$ (recovery) during time interval $\partial t$, equation (4) may be written as the system:

$$
\frac{\partial P_{1}}{\partial t}=-\alpha p_{1}+\beta p_{2} ; \frac{\partial P_{2}}{\partial t}=-\alpha p_{1}-\beta p_{2},
$$

Solving equation (4) with initial conditions $t=0, p_{1}=1$ (recovery), we get:

$$
\begin{aligned}
& p_{1}(t)=e^{-\lambda t}+\beta / \lambda\left(1-e^{-\lambda t}\right), \\
& p_{2}(t)=\beta / \lambda\left(1-e^{-\lambda t}\right),
\end{aligned}
$$

The two-stage model identifies state 1 (recovery) with the contact which is in the adhesion state, and state 2 (bonding failure) with the contact in the state of frictional sliding (6) and (7). Their interaction may be interpreted as the probability of the event when the contacts are in the said states or as a relative number of such contacts that are present in the system at every given moment of time $t$.

Therefore, estimating stationary and, consequently, ergodicity, and considering micro-contact models of the roughness model on the basis of expressions (6) and (7), we can see that the said equation has stationary solution when $t \rightarrow \infty$.

As the probability $p_{1}(t)$ is proportional to the number of contacts in the adhesion state (when elementary force of adhesion friction per one contact is considered), expression (6) shall be similar to the dependence of force of static friction $F_{s f r}$ (and friction coefficient $\mu$ ) on the duration of static contact, which has been established by I. V. Kragelskiy:

$$
F_{s f r}=F_{\infty}-\left(F_{\infty}-F_{0}\right) e^{-v t}=F_{0} e^{-v t}+F_{\infty}\left(1-e^{-v t}\right),
$$

here $F_{\infty}$ is the force of friction, that corresponds to the infinitely longstanding contact, $F_{0}$ is the force of friction at a contact of zero duration.

The probability interpretation of velocity dependence of the force of the sliding block pull-off from the surface of rotating disk that possesses mobility under certain conditions of the contact interaction is given in close relation to the twostage model, [12].

Hence, to estimate a relative number (probability) $P$ of constraints existing in the system and broken at the moment of their sliding from the surface of an absolutely elastic body at constant sliding velocity $v$, we can write:

$$
P=P_{01} e^{\alpha v}+P_{02}\left(1-e^{-\beta v}\right)
$$

here $P_{01}, P_{02}$ is the relative number of microroughnesses in every state when $v=0, v=\infty$, respectively; $\alpha, \beta$ are 
coefficients.

Comparing (8) and (6) and writing the expression for $\mu$ friction coefficient we get:

$$
\mu_{\infty}=\frac{\beta}{\lambda}=\frac{\beta}{\alpha+\beta} .
$$

Equation (7) is related to the force of friction of motion. If there is a number of model states with a big number of microcontacts, the equation has an oscillatory solution and, implicitly, a decrease in the force of friction must be observed.

Taking into account the difference in the height of microroughnesses, a discrete consequence of impulses of forces of friction shall be formed during motion, with arbitrary amplitudes, $\alpha v$, and moments of their appearance, $t v$; it corresponds to elementary displacements of interacting microscopic zones of sliding surfaces. On the basis of this system of impulses, which may be approximated, let us say, by $\Delta$-impulses, we can build a continuous random function $x(t)$ and a respective random process by averaging over large time intervals $\partial t^{\prime}$, which are larger then time intervals between individual elementary impulses:

$$
x(t)=\int \sum_{v} \alpha_{v} \Delta\left(t^{\prime}-t\right) \partial t^{\prime} .
$$

Physically, it corresponds to the fixation of resultant displacements (elastic wave) at large microsegments of surfaces of friction. In its turn, this random process which is averaged over time intervals, is approximated by Markov process, in other words, it is assumed that its distribution function $v\left(t_{1} x / t_{0} x_{0}\right)$ is described by Fokker Planck equation:

$$
\frac{\partial v}{\partial t}=\frac{\partial A\left(x_{1} t\right) v}{\partial x}+\frac{1}{2} \frac{\partial^{2} B\left(x_{1} t\right) v}{\partial x^{2}},
$$

here $A, B$ are coefficients.

Now let us introduce a conditional average $\bar{x}(t)$ over displacements, but for process (9), assuming that at the moment of time $t_{0}$ :

$$
\bar{x}=x_{0} ; \bar{x}(t)=\int_{-\infty}^{+\infty} x v\left(t_{1} x / t_{0} x_{0}\right) d x .
$$

We form a phenomenological equation of motion:

$$
\int_{-\infty}^{+\infty} A v d x=A\left(\overline{x_{1} t}\right)
$$

multiplying equation (9) by $x$ and integrating over $x$ within the limits from $-\infty$ to $\infty$, assuming that integrated terms become zero at infinity.

Equation (12) is in line with the standard equation of dynamics for a mass point, provided that:

$$
A\left(\overline{x_{1} t}\right)=a(t) \bar{x}+b(t) .
$$

Therefore, Fokker-Planck-Kolmogorov equation is equivalent to the stochastic equation of motion, that is, the use of the stochastic equation when describing the motion of effective volume of interaction which is in the adhesion state, as well as resolution of the stochastic force of reaction into an average and fluctuating components within the said framework for the purpose of physical-statistical interpretation, seems to be clear and acceptable. In this case the effective mass may be considered as a proportionality factor included into $A\left(\overline{x_{1} t}\right)$. In view of the existing relationship between equations (9) and (12) we formulate the idea of the exponential energy distribution for the system of microcontacts in the adhesion state.

As the stationary solution of equation (10) exists, $\partial v / \partial t=0$, then:

$$
j=-A v+\frac{1}{2} \frac{\partial B v}{\partial x}=\text { const }
$$

The stationary flow $j$ is supposed to be zero at the border of $x$ variation range, then, after integration:

$$
v(x)=\frac{C}{B(x)} e^{\int_{0}^{x} \frac{A(S)}{2 B(S)} d s},
$$

here $C$ is the constant, whose value is to be found from normalization condition. If at constant $A$ and $B$ we assume that:

$$
\int_{0}^{x} \frac{A(S)}{2 B(S)} d S=\frac{A x}{2 B}=-\frac{\Delta E}{\Theta}
$$

here $\Delta E$ is the energy of the contact in the adhesion state; $\Theta$ is the modulus of canonic distribution, then:

$$
v(x)=\text { conste }^{-\frac{\Delta E}{\Theta}} .
$$

Distribution (16) seems to be equivalent to (15), however, not for a system of micro-displacements, which is described by equation (11), but for a system of progressive waves induced by them, which transforms external friction caused by molecular and mechanical components at micro-contact, into internal friction.

The numerical value of the constant is determined by physical and mechanical properties and the structure of tribosystem elements, that cause the transfer of the most part of energy generated during local dynamic interaction, by deformation waves. It is logical to assume the existence of a certain critical value of velocity, $v(x)$, when the said balance becomes disturbed and the material instantly destroys. This hypothesis is confirmed by the studies of the mechanism of energy transfer at shock loading of materials due to propagation of waves of elastic and plastic deformations [25]. While studying the propagation of shock waves in continuous media, the authors used the hydrodynamic theory; pursuant to this theory the shock front is interpreted as the surface of jump of the function that describes the motion of particles before and behind the shock front. To describe the 
behavior of materials in the said conditions, equations of momentum change, balance of mass and energy flow when the surface of jump of function moves in a heterogeneous continuous medium, were used. In this case, the dependence of critical velocity $v_{\kappa p}$ on energy spent for deformation of a unit volume of the material $E_{c o l}$ may be approximated by the expression:

$$
v_{c r}=\left(2 \mathrm{kE}_{\mathrm{col}} / \rho\right)^{\frac{1}{2}}
$$

here $\mathrm{k}$ is the proportionality factor; $\rho$ is the strain with account of "viscous" losses (internal friction).

Expression (17) in view of its physical interpretation, is close to expression (16).

The aforesaid calculations apply to longitudinal waves because elastic oscillations have a transverse component in addition to longitudinal waves. As to oscillations that propagate into the internal zone of triboelements, while taking into account that in case of elastic oscillations the value of local strains in the whole volume must be final, then every component of these oscillations is a standing wave [26]. The displacement distribution forms zones of volume that oscillate in opposite phase, as a result the acting strains and, consequently, tribosystem wear becomes zero.

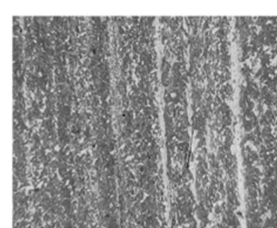

a

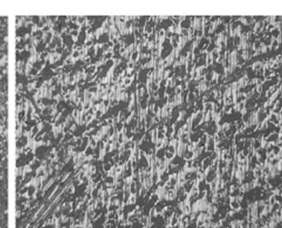

$\mathrm{b}$

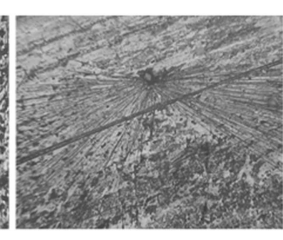

$\mathrm{c}$
Fig. 4. Surface fractography of triboelements made of bronze ВБ23НЦ $\times 250$ : $a$ - the basic tribosystem; $b$ - at program loading (impulse loading equals $1400 \mathrm{~N}$ ); c-at program loading (impulse loading equals $1800 \mathrm{~N}$ ).

In our opinion, the condition of anomalously low friction and wear consists in the equality of energy components of molecular-mechanical and wave components of forces of friction. If this equality is violated (Fig. 4, c), then defects are accumulated in the subsurface zone and wear particles are formed; as to the wear mechanism, it must be studied with more details using up-to-date methods of physics of metals and modern physical methods. The further studies of anomalously low friction of the tribosystem under investigation at various values of increase in the impulse loading value by two grades up to $1800 \mathrm{~N}$ (Fig. 2-4, c) at the final stage of the program loading, confirmed this hypothesis.

In this case a tribosystem passes into the zone of negative friction up to the friction coefficient value of $\mu=-0,035$ with further stabilization at $\mu=-0,009$ (Fig. 2), which is caused by disbalance between absolute values of molecular-kinetic and wave components of forces of friction. The physical aspects of this transition may be explained only from the standpoint of the non-equilibrium thermodynamics after the detailed study of microstructural changes of elements of the given tribosystem along the depth of the surface layer, applying up-to-date physical theories [27], that allow to have a new vision of the effect of energy distribution symmetry on the mechanism of its transfer (theory of super-strings).
In case of negative friction, a significant wear rate is observed; it is registered on the basis of the parameters of acoustic emission. Physics of this kind of wear is related to the internal processes of energy exchange in the subsurface zone of the triboelement. Coarse wear particles on the friction surface appear like micro-explosions, Fig. 4, c.

Therefore, in accordance with conclusion given in published work [13] by A. I. Veinik, when the force of friction and wear represented in Fig. 2-4, b, become zero, the violation of the law of energy conservation is not observed. However, if the energy balance between molecular-mechanical and wave components of the force of friction is disrupt, which is observed in case of negative friction, the wear that corresponds to the absolutely different physical mechanism shall be observed. Wear particles, in accordance with fractographic studies, are formed in the subsurface layer, and they break the undamaged surface layer, and also, a relief trace which is typical for friction and aligned along motion direction, is not formed, when a wear particle leaves the contact zone. In this case, the possible destructing mechanism of wear may be a non-balanced internal friction. However, this statement must be proved by more detailed studies.

\section{Conclusions}

Hence, in addition to molecular and mechanical components of the force of friction at anomalously low wear a wave component appears, and in this case the effect of this component prevails. In essence, under conditions of anomalously low friction and wear, the phenomenon of Gray's paradox is realized on the higher level of selforganization. In our opinion, the transit of the tribosystem from the normal to anomalously low friction using the program loading generates the special kind of wave resonance, which appears in the kinetic nanofield together with progressive wave formation. This effect does not appear under the conditions of equilibrium self-organization, as the principles of the thermodynamics of equilibrium systems and, in particular, formation of the positive hardness gradient (Rehbinder effect), impede it.

Summarizing the undertaken study we make the final conclusion that the hypothesis concerning the existence of anomalously low and even negative friction proposed by A. I. Veinik, is true, it is confirmed experimentally and has a great practical significance. For instance, the transit of the tribosystem to zero friction under conditions of program loading for steel-bronze tribosystem increases its wear resistance by up to 140 times comparing with the basic tribosystem, Fig. 2-4, a. In this case, the wear takes place at the initial stage of the program loading (wearing-in) and stops at the final stage of the impulse loading, which is confirmed by the results of observation of the wear rate using the acoustic emission method.

\section{References}

[1] Kostetskiy B. I. Surface strength of materials at friction [Text] / B. I. Kostetskiy, I. G. Nosovskiy, A. K. Karaulov, et al. Kiev: Tekhnika, 1976.-296 p. 
[2] Kragelskiy I. V. Principles of friction and wear calculations [Text] / I. V. Kragelskiy, M. N. Dobychin, V. S. Kombalov. Moscow: Mashinostroienie, 1977.-526 p.

[3] Stadnichenko V. N. Synergy concept of self-organization in tribologic systems at heat flow control [Text] / V. N. Stadnichenko, O. N. Troshin // Bulletin of "KhPI" Technical University. Collection of research papers: Technologies in machine building. Kharkiv: National Technical University "KhPI"- 2007. - No. 17 - Pp. 49-62.

[4] Tribology. Physical principles, mechanics and engineering applications [Text] / I. P. Berkovich, D. G. Gromakovskiy; under the editorship of D. G. Gromakovskiy - Samara: Samara State Technical University, 2000-268 p.

[5] Zaporozhets V. V. Mechanism of energy dissipation at metalceramic layer friction in technologies of tribotechnical recovery of machine and mechanism elements [Text] / V. V. Zaporozhets, V. N. Stadnichenko, O. M. Troshin // Weapon systems and military machinery. - Kharkiv: 2010. - No. 2(22). - P. 113-118.

[6] Troshin O. N. Methodological aspects of non-equilibrium selforganization of tribosystems [Text] / O. N. Troshin, V. N. Stadnichenko, N. G. Stadnichenko, et al. // Weapon inventory and military machinery. - Kharkiv: 2014. - No. 1(37). - P. 233-241.

[7] Tribotechnical reference guide, in 3 vol. Vol. 1. Theoretical fundamentals [Text] / Under the general editorship of $\mathrm{M}$. Khebda, A. V. Chichinadze. - Moscow: Mashinostroyenie, 1989. $-400 \mathrm{p}$.

[8] Phenomenon of anomalously low friction in vacuum [Text] / E. A. Dukhovskiy, V. S. Onishchenko, A. N. Ponomariov, A. A. Silin, V. L. Talrose, No. 121 with priority as of September $16,1969$.

[9] Stadnichenko V. N. Prediction of tribosystems life-time when they operate in the nanowearing mode, using the method of acoustic emission [Text] // V. N. Stadnichenko, O. N. Troshin, A. V. Piymak et al. // Collection of research papers of Kharkiv University of Air Forces. - Kharkiv: 2010. - No. 4(26). - P. 41-48.

[10] Patent 2397101 C1 Russian Federation, МПК В63B 1/36 (2006.01) Apparatus which increases the rate of sailing of a vessel [Text] / Dziuba A. F.; patent holder: A. F. Dziuba. No. 2009121794/11; appl. 08.06.2009; publ. 20.08.2010, bul. No. 23.

[11] Patent 41896 Ukraine, МПК В23P 6/00, C23C 24/00 C10M 125/30(2009.01) Method of recovery of tribosystem friction surfaces without disassembling [Text] / V. A. Voytov, Stadnichenko V. M., Stadnichenko M. G., Bilyk A. P.; applicant and patent holder Kharkiv National Technical University of Agriculture named after P. Vasylenko u200901117; appl. 12.02.2009; publ. 10.06.2009, bul. No. 11 .

[12] Zaporozhets V. V. Mechanism of energy dissipation at metalceramic layer friction in technologies of tribotechnical recovery of machine and mechanism elements [Text] V. V. Zaporozhets, V. M. Stadnichenko, O. M. Troshin // Weapon inventory and military machinery. - Kharkiv: 2010. - No. 2(22). - P. 113-118.

[13] Veinik A. I. Thermodynamic couple [Text] / A. I. Beinik Minsk: Nauka i tekhnika, 1973-284 p.
[14] Stadnichenko V. M. Methodology of contact interaction processes control on the basis of acoustic-emission effects in tribotechnical systems [Text]: synopsis of Doctor of Engineering Science thesis / V. M. Stadnichenko, National Aviation University. - Kyiv 2014. - 40 p.

[15] Gindin I. A. Physics of program hardening [Text] I. A. Gindin, I. M. Nekliudov, - Kiev: Naukova dumka, 1979.-184 p.

[16] Vasiliev A. S. Targeted formation of properties of engineering products [Text] / Vasiliev A. S. et al. - Moscow: Mashinostroyenie, 2005. - $384 \mathrm{p}$.

[17] Nikolas G. Challenge knowledge [Text] / G. Nikolas, I. Prigozhin. Trans. From English - Moscow: Mir, 1990-344 p.

[18] Zaporozhets V. V. Theoretical and experimental fundamentals of acoustic-emission identification of wear mechanisms and prediction of tribosystems life-time [Text]// V. V. Zaporozhets, V. N. Stadnichenko, O. N. Troshin // Tribology problems. Khmelnytskiy: 2013. - No. 1(67) - P. 89-98.

[19] Stadnichenko V. N. Classification of nanowear types with regard to the dissipation coefficient of external energy supplied to the tribosystem [Text] / V. N. Stadnichenko, O. N. Troshin, N. G. Stadnichenko et al. // Collection of research papers of Kharkiv University of Air Forces. - Kharkiv: 2010. - No. 1(27). - P. 51-61.

[20] Baranov A. V. Self-organization of tribosystems under conditions of metals boundary friction $[$ Text $]$ / A. V. Baranov, V. A. Vagner, S. V. Tarasevich et al. // Polzunov bulletin. 2009. - No. 1-2. - P. 155-158.

[21] Pogodayev L. I. Structure-energy models of materials behavior (reliability) at impulse loading / Friction, wear, lubrication. - 2013. Volume 15, No. 57 [Electronic resource]. - Access mode: http://www.tribo.ru.

[22] Ruban I. V. Automation of processes of laboratory and stand trials in triboengineering [Text] / I. V. Runab, O. N. Troshin, S. V. Smirnov et al. // Weapon systems and military machinery. - Kharkiv: 201-. - No. 3(23). - P. 150-153.

[23] Baranov A. V. Prediction method and the way of life time increase for wearable movable conjugation of machine elements [Text]: $\mathrm{PhD}$ (Engineering science) thesis / A. V. Baranov; Leningrad Politechnic Institute. - Leningrad: 1988.$175 \mathrm{p}$.

[24] Poliakov S. A. Theoretical analysis of principal mechanisms of tribosystems evolution at selective transition [Text] / S. A. Poliakov // Service life of machine friction members. Moscow: 1988. Num. 3.-P. 3-27.

[25] Yezhov Yu. Ye. About critical rate of loading for heterogeneous material under erosion conditions / Friction, wear, lubrication. // Yu. Ye. Yezhov, L. I. Pogodayev, Yu. T. Borshchevskiy. Volume 15, No. 57 [Electronic resource]. Access mode: http://www.tribo.ru.

[26] Goloskokov Ye. G. Elastic and acoustic problems of dynamics of three-layer constructions [Text] / Ye. G. Goloskokov, S. N. Beshenkov. - Kharkov: Vyshcha shkola, 1980.-120 p.

[27] "Introduction to Superstring Theory", E. Kiritsis, Leuven Univ. Press, 1998 (Leuven Notes in Mathematical and Theoretical Physics, Vol. 9). 